\title{
Pour une théorie périphérique et/ou amoureuse : lectures d'Agamben, Derrida, Rancière
}

\author{
FRANCESCA MANZARI
}

\begin{abstract}
For a Peripheral Theory in Love: Reading Agamben, Derrida, Rancière. The introduction of Giorgio Agamben's book entitled Stanzas, Word and Phantasm in Western Culture is about the relationship of philosophy and poetry to knowledge in Western culture. The stanza is "the essential nucleus" of Tuscan poetry in the thirteenth century. It is actually an invention of Tuscan poets who call stanzas the parts that compose every canzone. Stanza is a word for chamber in Tuscan dialect as well as in Italian. Agamben points out that what makes possible its poetical existence is the fact that a stanza is a topos outopos, a topos which contains its own negation: it is the reality of unreality. Agamben's thesis is that Western culture has forgotten the unitary status the Western word had until the thirteenth century. The thirteenth century could still conceive poetic activity as a philosophical one and then Western culture has known a separation between two poles that define knowledge and word. This paper aims to investigate the relationship between knowledge and words in Derrida, Rancière and Agamben.
\end{abstract}

Keywords: Giorgio Agamben; Jacques Derrida; Jaques Rancière; stanza; théorie amoureuse

Dans l'introduction d'un ouvrage intitulé Stanze : Parole et fantasme dans la culture occidentale, Giorgio Agamben esquisse la forme d'une double trajectoire, critique et théorique : il s'agit d'un côté de définir le rôle de la critique dans l'histoire de la pensée occidentale et de l'autre de fonder une théorie à partir d'un positionnement critique. Dès les premières lignes de son introduction, Agamben commence par rappeler qu'un ouvrage de critique produit une attente de résultat : « tout au moins des thèses à étayer et, comme on dit, des hypothèses de travail ». La direction est annoncée d'emblée, l'ouvrage se fonde sur une critique pour proposer une théorie. Il s'agit alors de revenir sur la définition de la pratique de la critique : « lorsqu'il fait son apparition dans le vocabulaire de la philosophie occidentale, le mot critique signifie plutôt enquête sur les limites de la connaissance, c'est-à-dire sur ce qui ne peut être précisément ni posé ni saisi » (1981: 7).

Dans un séminaire intitulé Théorie et pratique, Jacques Derrida propose une définition du texte philosophique qui constitue un vis-à-vis intéressant pour la 
Pour une théorie périphérique et/ou amoureuse : lectures d'Agamben, Derrida, Rancière

description qu'Agamben donne du fonctionnement de la critique. Derrida vise l'explicitation du rapport théorie/pratique et affirme d'emblée que la pratique de la philosophie devrait toujours être révolutionnaire et en ceci elle aurait un rapport à la théorie. La question traitée a aussi à voir avec la problématique du performatif en ceci que seulement en affrontant l'injonction performative, on se rapproche de ce qu'est une pratique révolutionnaire de la philosophie,

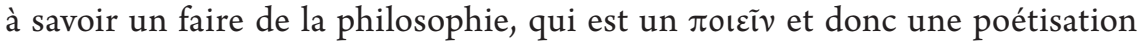
de la philosophie. C'est dans la révolution morpho-centrique du texte philosophique, semblable pour Derrida à celle que Mallarmé opère en poésie, qu'on est le plus près de la compréhension de ce que le philosophe entend par pratique révolutionnaire de la philosophie. Celle-ci est toujours contextualisée, rappelle Derrida. Et pour comprendre ce qu'est un contexte, il faut dépasser le sens courant du mot texte. Derrida donne alors une définition du texte qui revient à plusieurs reprises dans ses écrits : « un réseau de rapports à l'autre, de différences et de traces de différences dans un procès de différence ». Toutefois, ce n'est pas celle qu'il faudra retenir pour le contexte de la pratique révolutionnaire. Texte est ici à entendre comme un lieu « comprenant tout ce que la philosophie et le langage traditionnel qui lui est lié appellent l'histoire, l'économie, la politique, etc. » (2017:27). Ce texte incorporant son propre objet, son contenu, pose de son bord interne la question de son débordement. Derrida se réfère à une « question » récurrente dans le séminaire, celle qui est posée sur le rapport $\ll$ du couple théorie/pratique $\gg$. Cette interrogation fait immédiatement trembler l'assurance du $\theta \varepsilon \omega \rho \varepsilon \tilde{\varepsilon} v$, de la contemplatio, du concevoir. De son bord intérieur, la philosophie pose une question qui est déjà un faire. C’est une nouvelle façon de faire de la philosophie et c'est une pratique révolutionnaire, où le mot pratique n'est pas simplement accompagné par l'attribut de révolutionnaire, mais travaillé de l'intérieur (c'est encore un bord interne). La pratique révolutionnaire est d'emblée débordante.

Ainsi, la définition donnée par Agamben de la critique comme « enquête sur les limites de la connaissance $\gg(1981: 7)$ peut alors être lue à partir de la problématique annoncée par Derrida, celle de la philosophie comme rapport entre une théorie et une pratique. Il ne s'agit pas seulement d'entendre par là la critique comme enquête autour des limites de la connaissance, mais d'entendre ici la préposition sur comme l'introduction d'un complément de lieu. Il faudra dès lors entendre la critique comme une enquête menée littéralement à l'endroit des limites de la connaissance ou, pour le dire avec Derrida, à l'endroit du débordement de celle-ci et dans ce sens, la pratique de la critique est alors une activité philosophique et bien plus, une activité philosophique authentique parce que révolutionnaire, toujours déjà d'emblée prête, du bord de sa pratique, à devenir une théorie nouvelle et renouvelante. La question pourrait 
alors être ici posée de savoir si toute critique peut être considérée comme une activité philosophique. Pour répondre avec Agamben, seulement les rares ouvrages de la tradition occidentale qui incluent leur propre négation, comme L'Origine du drame tragique allemand de Walter Benjamin, où l'objet de l'étude se dérobe sans cesse à son propre saisissement, peuvent recevoir l'attribut de « critique » : «Comme toute quête authentique la quête critique consiste, non point à retrouver son objet, mais à assurer les conditions de son inaccessibilité » (1981: 8-9).

Jusqu'ici, auront été évoquées au moins trois catégories qui permettent le classement d'une partie significative de la production intellectuelle occidentale. La définition de la critique d'un côté, qui fonde la réflexion d'Agamben, la théorie qui est pour Derrida une des deux régions dans lesquelles est partagées l'activité philosophique : la théorie d'un côté et la pratique de l'autre. Ici la pratique a une fonction métonymique et tient la critique et la théorie ensemble au sein d'une activité que l'on peut encore appeler philosophique. En d'autres mots, une critique authentique au sens d'Agamben, est une pratique textuelle qui a pour objet le saisissement de ce qui s'échappe. Or ce qui par excellence échappe dans la tradition de la pensée occidentale est l'étendue de l'intellect et c'est de cette difficulté que naît le $\theta \varepsilon \omega \rho \varepsilon \tilde{\imath} v$. Avec Agamben, il est possible d'ajouter aux trois productions textuelles jusqu'ici mentionnées une quatrième, celle de la poésie. Le philosophe évoque le groupe de Iéna et son projet de « poésie universelle progressive » qui abolit la distinction entre poésie et disciplines critico-philologiques» (1981 : 7-8). Les trois premières catégories textuelles, la critique, la théorie, la philosophie sont alors pensées dans un agencement avec la poésie.

Que la question soit pour Agamben, comme pour Derrida dans le séminaire Théorie et pratique, une question de bord et de débordement, cela est confirmé par la conclusion de l'introduction de Stanze. La stanza - qui fait l'objet de l'ouvrage - est un lieu, la chambre de la poésie, plus précisément cela viendrait de la cambra, mot occitan pour chambre, du château dans lequel la dame s'entretient secrètement avec le troubadour pour le soumettre à l'assag : la dame se couche nue, à la lumière d'une lampe, à côté du troubadour qui doit maîtriser la distance entre son corps et celui de l'aimée, prouvant ainsi l'authenticité de son sentiment amoureux. La dame touchée par la valeur du poète aime en retour et c'est sous un senhal, un nom écran, qu'elle fera l'objet de compositions chantées devant la cour.

Il s'agit de rester à une distance mesurée, de ne pas céder à la tentation sexuelle. Cette résistance est l'un des traits distinctifs de la poétique du trobar, qui trouve son origine dans une forme de plaisir qui est bien connue et codifiée au Moyen Âge : la delectatio morosa, un « plaisir dans lequel on s'attarde » selon 
Pour une théorie périphérique et/ou amoureuse : lectures d'Agamben, Derrida, Rancière

l'expression de Charles Baladier. La delectatio morosa est une forme de destin du désir lorsqu'il est dirigé sur une réalité absente, « cette brûlure hallucinante qui consiste dans la recherche d'un être qu'on ne possède pas encore ou qu'on ne pourra posséder » (2001: 137). L'inappropriabilité de l'être absent devient en soi un plaisir. Cela consiste en un « arrêt sur image », complaisance intensément éprouvée dans la durée du fantasme. Jacques Lacan s’y réfère lorsqu'il écrit que « le fantasme fait le plaisir propre au désir » (1963: 299).

Le désir de l'inappropriable que le troubadour chérit jusqu'à son paroxysme ne devrait être assouvi que pour rendre possible un état de joi ${ }^{1}$ qui est un effet tout à la fois de démesure et de mesure, puisque son débordement entraînerait la mélancolie et donc l'impossibilité de composer. Le désir de la dame est plus authentiquement un désir de la poésie, désir d'atteindre ce qui, en tout état de cause, ne peut qu'être en fuite, la beauté du fantasme, de la pensée, du poème. Or la poésie toscane du XIII siècle, plus précisément les compositions des poètes du Doux Style Nouveau, Dante, Cavalcanti, Guinizzelli, donnent une forme plus appropriée à cette intuition du trobar clus qui lie le fantasme et l'intellect à la poésie : les poètes toscans du XIII e siècle définissent l'amour comme une pensée immodérée. Le poème est la forme donnée au débordement de la pensée du poète amoureux. Si Agamben consacre son ouvrage à la stanza de la canzone toscane, c'est parce que ce lieu de la poésie occidentale que le philosophe définit comme topos outopos garde en soi la trace du rapport que la poésie entretient avec la philosophie en Occident. Les poètes du Doux Style Nouveau abandonnent l'accompagnement musical qui était un trait constitutif des cansos des troubadours. Le retrait de la musique correspond à l'entrée de la philosophie dans la canzone. La légèreté de la musique cède la place à un alourdissement qui n'est pas seulement technique - une stanza est plus lourde en nombre de vers qu'une cobla ${ }^{2}$-, mais également un alourdissement du contenu. La stanza esquisse le mouvement de l'immoderata cogitatio et la canzone porte un contenu philosophique. La beauté des stanze tient alors à l'équilibre fragile du saisissement de ce qui est toujours déjà en cours de débordement.

Sur un plan théorique, la forme des stanze indique une voie au critique littéraire. Ce qu'il faudra désormais chercher dans un texte n'est pas là, le texte critique dit cette absence, toute lecture critique la relèvera et la renouvellera. Par ailleurs, si le renouvellement est le premier axiome du trobar, la difficulté du geste critique, qui est toujours mesuré et à la recherche d'un équilibre impossible, puisqu' il tient et s'exerce sur le bord du connaissable, est depuis le Moyen Âge profondément lié au surgissement de la composition poétique.

L'union indissoluble de la joie et de la souffrance.

2 Strophe de la canso. 
La critique comme enquête sur les limites de la connaissance et donc comme activité pratiquée à la périphérie de la connaissance est comparable à la pratique de la philosophie telle que Derrida la décrit dans Marges de la philosophie lorsqu'il écrit : «Tympaniser - la philosophie » (1972: I), c'est-à-dire la soumettre à une fictionnalisation, non pas au sens aristotélicien, mais au sens que le mot fiction acquiert dans le régime esthétique. Dans la partie intitulée « des modes de la fiction $\gg$, dans Le partage $d u$ sensible, Jacques Rancière rappelle l'enjeu de la Poétique d'Aristote : «soustraire les formes de la mimesis poétique au soupçon platonicien sur la consistance et la destination des images $\gg$, , $<$ proclamer que l'agencement d'action du poème n'est pas la fabrication d'un simulacre $\gg$, mais « un jeu de savoir qui s'exerce dans un espace-temps déterminé », parce que « feindre [...] n'est pas proposer des leurres », mais « élaborer des structures intelligibles $\gg$ (Rancière 2000 : 55-56). Les fictions dont est faite la poésie sont « des agencements entre des actes ». C'est donc l'agencement qui est au cœur de la définition de la fiction. Cela confère à la poésie un caractère de supériorité, si on la compare à l'histoire : la fiction rationnalise, là où l'histoire et sa science ne peuvent le faire (voir Rancière 2000 : 59).

Ce clair partage est interrogé, le jeu redistribué, par la révolution esthétique qui d'un côté « brouille les frontières entre la raison des faits et celles des fictions », et accorde, de l'autre, « un nouveau mode de rationalité » à la science historique. L'âge romantique « enfonce le langage dans la matérialité des traits par lesquels le monde historique et social se rend visible à lui-même $\gg$. Une nouvelle fictionnalité peut alors être définie : « la nouvelle manière de raconter des histoires, qui est d'abord une manière d'affecter du sens à l'univers "empirique" des actions obscures et des objets quelconques $\gg$ (Rancière 2000 : 56-59).

L'enchaînement causal d'Aristote, motivé par la nécessité et la vraisemblance, est remplacé par un agencement de signes, qui ne sont pas une marque d'auto-référentialité solitaire du langage, mais « l'identification des modes de la construction fictionnelle à ceux d'une lecture des signes écrits sur la configuration d'un lieu, d'un groupe, d'un mur, d'un vêtement, d'un visage ». La souveraineté esthétique de la littérature « forge » une « rationalité nouvelle du banal et de l'obscur qui s'oppose aux grands agencements aristotéliciens et deviendra la nouvelle rationalité de l'histoire de la vie matérielle opposée aux histoires des grands faits et des grands personnages ». Naît ainsi une histoire poétique articulant une réalité qui est parsemée de traces poétiques et un « artificialisme » qui échafaude « des machines de compréhension complexes ». Cela parce que « le réel doit être fictionné pour être pensé » (2000:57-61). Ce qui ne veut pas dire que « tout est fiction », mais que la fiction à l'âge esthétique modalise un lien « entre présentation des faits et formes d'intelligibilité », ce qui ne permet plus de penser la raison de la fiction en termes aristotéliciens. 
Pour une théorie périphérique et/ou amoureuse : lectures d'Agamben, Derrida, Rancière

Il ne s'agit plus seulement d'écrire ce qui pourrait être, mais ce qui pourrait, aurait pu être, ce qui a été, ensemble ou séparément selon une disposition, une fabrication, qui implique à la fois une idée de la « raison » de la fiction comme « empirisme » de l'histoire d'une « fabrication », une fiction est toujours une histoire d'agencements, et de «l'empirisme de l'histoire » comme une potentialité fictionnelle. À bien vouloir regarder, il est toujours possible de trouver, à l'infini, une voie de construction fictionnelle nouvelle dans l'observation empirique des choses. Ce brouillage opéré par l'âge romantique est politique pour Rancière : «la politique et l'art, comme les savoirs, construisent des “fictions", c'est-à-dire des réagencements matériels des signes et des images, des rapports entre ce qu'on voit et ce qu'on dit, entre ce qu'on fait et ce qu'on peut faire » (2000 : 62).

Politique, art et savoir construisent donc des fictions. Elles ne sont pas les mêmes, bien entendu, mais certaines conséquences peuvent être déduites de la thèse bien connue de Rancière. L'agencement rationnel et donc métaphysique, opposé à l'empirique chez Aristote, devient, dans le régime esthétique, matériel. Cette matérialité est l'effet rendu par l'entrée des objets comme signes, dans la fiction. Leur fonction est de restituer un corps à une construction qui ne demeurait que dans le métaphysique : ce qui pourrait être, mais n'est pas. Les objets matériels, le joujou du pauvre de Baudelaire, la toile et l'habit dans Le Capital, matérialisent la fiction.

Mais Rancière va plus loin, il dit que les signes qui composent la fiction sont devenus matériels. C'est bien cela que Derrida entend en formulant le projet de tympaniser la philosophie. Pour Jacques Derrida, bien avant 1993, année de la parution de Spectres de Marx, la question du marxisme et du matérialisme dialectique ${ }^{3}$ est liée d'un côté à une pensée du bord et de l'autre à son dépassement, à une matérialisation de la philosophie et donc à ses ressorts fictionnels.

En 1975-1976, lors de la première séance du séminaire de l'ENS intitulé Théorie et pratique, Derrida dit, au sujet de la critique adressée par Marx à Feuerbach, qu'elle atteint « tout ce qui, dans l'histoire de la philosophie, place l'attitude théorique au-dessus de la pratique et y voit l'accomplissement authentique ou suprême de l'homme, c'est-à-dire peut-être la quasi-totalité de la philosophie $\gg$. Si Marx atteint ainsi toute la philosophie, Derrida laisse ouverte « la question de savoir si cette critique est encore philosophique, appartient à quelque chose comme la philosophie, la supprime ou la déborde $\gg(2017: 24)$.

La réponse donnée dans le séminaire, dès la cinquième séance, et grâce à la lecture d'Althusser, c'est qu'il existe une philosophie marxiste ou un projet

3 Derrida emploie l'expression entre guillemets. Celle-ci n'apparait jamais chez Marx. Engels l'utilise seulement en 1886. 
philosophique marxiste. La pensée de Marx n’a donc pas encore débordé la limite de la philosophie, mais elle est toujours prête à le faire. La chose nommée « bord de la philosophie » a du moins à voir avec la pertinence du couple théorie/pratique parce que c'est depuis le bord interne de la pensée de Marx que cette opposition ou cet accouplement théorie/pratique peut être pensé.

La cinquième séance rétablit donc la place du marxisme à l'intérieur de la philosophie. Toutefois, cette place occupée par le matérialisme dialectique à l'intérieur de la philosophie est une région frontalière puisque c'est le lieu où il soit possible d'entrevoir une région qui ne soit pas seulement de la philosophie, où il est possible de concevoir un dépassement de la philosophie.

La métaphore du champ est invoquée, elle aussi, dès la première séance et toujours à propos de la Onzième Thèse sur Feuerbach. Elle n'est pas opérationnelle, dit Derrida. Elle est trop usée. Derrida rappelle alors que les thèses de Marx sont des textes, fonctionnent comme des textes. Il cite d'abord la Troisième Thèse : « la coïncidence du changement des circonstances et de l'activité humaine ou de l'auto-transformation ne peut être saisie et rationnellement comprise qu'en tant que pratique révolutionnaire $\gg(2017: 25)$.

La pratique de Derrida, son faire, dans le séminaire illustre parfaitement, ou presque, ce que Rancière dit dans Le partage du sensible, et plus tard, dans Les bords de la fiction, au sujet du régime esthétique. Derrida matérialise, fictionnalise, le texte de Marx, le fait devenir un lieu. Un lieu qu'on regarde ou à partir duquel on regarde et dans lequel on reconnaît des parts et des places à prendre, des lieux dans lesquels agir pour être ou non des agents révolutionnaires. Derrida le dit, dans de la cinquième séance du séminaire : la philosophie marxiste, « existante ou à venir, [...] doit déterminer la place, le site et les rapports du théorique et du pratique $\gg(2017: 102)$. Bien que théorique et pratique soient ici des philosophèmes, la place ne l'est pas.

Le texte philosophique à même de transformer la pratique de la philosophie et de la rendre nouvelle n'est pas un texte qui aurait un contenu nouveau, mais un texte qui serait fait, écrit, d'une manière nouvelle. Derrida dit : « ce n'est plus le contenu qui est renouvelé mais la pratique, la manière de faire, le faire qui transformerait jusqu'à l'essence de la philosophie [...] qui est, elle, encore un philosophème à transformer $\gg(2017: 102)$.

Nous comprenons mieux alors la réponse de Derrida à Aijaz Ahmad qui, dans Marx er Sons, s'en prend au ton de Spectres de Marx, ou alors à Eagleton qui dénonce le langage poétique de Derrida. Déjà en 1975, Derrida définissait la fidélité à Marx comme une forme d'écriture du texte philosophique : la seule pratique philosophique digne d'être considérée comme révolutionnaire.

Le séminaire Théorie et pratique permet aussi de mieux comprendre, rétrospectivement, le passage de Marx \& Sons sur le «perverformatif », mot que 
Pour une théorie périphérique et/ou amoureuse : lectures d'Agamben, Derrida, Rancière

Derrida avait employé en 1979, dans La carte postale. Derrida revient sur la question posée dans Spectres de Marx au sujet de la nécessité de la re-politisation et il dit : « Dans Spectres de Marx, comme dans tous mes textes, depuis vingt-cinq ans au moins, la prise en compte de la dimension performative (non seulement du langage au sens étroit mais de ce que j'appelle trace et écriture) aura partout déterminé et surdéterminé toutes mes argumentations » (Derrida $2002: 27$ ).

Derrida le dit dans la première séance du séminaire de 1975 : la question traitée a aussi à voir avec la problématique du performatif en ceci que seulement à vouloir affronter l'injonction performative, on se rapproche de ce qu'est une pratique révolutionnaire de la philosophie, à savoir un faire de la philosophie,

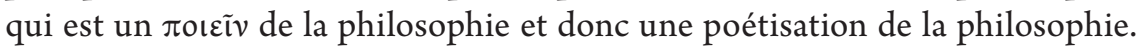
C'est dans la révolution morpho-centrique du texte philosophique qu'on est le plus près du projet marxiste, au plus près d'une philosophie révolutionnaire.

Ainsi, à l'encontre de ce que les détracteurs marxistes de Derrida auront pu dire, dans Ghostly Demarcations par exemple, très tôt, Derrida aura été marxiste, son écriture aura toujours été marxiste : lorsqu'elle se glisse entre les objets-mots poèmes de Ponge, lorsqu'elle s'occupe des objets dans la poésie de Baudelaire, lorsqu'elle tend l'oreille à la musique de l'écriture de Joyce, lorsqu'elle joue avec le nom de Hegel dans Glas. L'écriture de Derrida aura toujours tendu vers une fictionnalisation du philosophique et ainsi faisant elle aura été fidèle à Marx.

Précisément, Derrida lisant Marx fait ce qu'il a la compétence de faire selon la place qui lui est assignée dans la société, à savoir la place du philosophe. Il écrit donc des textes selon une praxis révolutionnaire et donnant à voir surtout le lieu de la lisière entre le théorique et le pratique, ce que fait Hegel et ce que fait Genet, lire Hegel à partir de Genet, lire Genet à partir de Hegel. Ce sont précisément les questions posées dans le texte de Glas. Loin d'être un brouillage des parts et des places, une pratique révolutionnaire de la philosophie ne fait que questionner les lieux de débordement (voir Derrida 1974 : 2-3).

Les colonnes de Glas sont des « réagencements matériels des signes et des images, des rapports entre ce qu'on voit et ce qu'on dit, entre ce qu'on fait et ce qu'on peut faire », selon le lieu institutionnel, philosophique ou littéraire, à partir duquel les énoncés qui les composent « font effet dans le réel ». C’est justement Rancière qui, dans Le partage du sensible, rappelle que « les énoncés politiques ou littéraires font effet dans le réel » (2002:62). Qu'en est-il des énoncés philosophiques ? Font-ils effet ? Font-ils de l'effet ? Et le fait d'en faire est-il une condition nécessaire pour qu'ils partagent du sensible ? Il me semble que depuis toujours, c'est l'implication même, la praxis même de l'écriture de Derrida. À vouloir mesurer ce qu'elle fait, son degré d'implication dans le faut 
le faire, on peut assurément dire qu'elle fait, entre autres, de l'effet et qu'à ce titre elle s'inscrit dans le partage du sensible.

L'objet de la Première Thèse sur Feuerbach reproche au premier matérialisme « d'avoir saisi 1' "objet" [...], 1' "effectivité" [...] et la "sensibilité" [...] sous la seule forme de l'“Objet" (Objekt) ou de l'intuition, et non pas en tant que "praxis” ou qu'“activité humaine sensible”, c'est-à-dire subjectivement » (Derrida 2017 : 23). Où le subjectivement est le mode de l'« activité humaine sensible » et définit la praxis qui devient une activité objective. Cette activité objective, cette activité humaine comme objet à saisir a une effectivité. L'activité humaine sensible est donc mesurée à son degré de production d'un effet réel dans le réel. Pour que l'écriture philosophique soit matérialisée, il lui faut donc produire de l'effet. En d'autres mots, la philosophie marxiste existante ou à venir est reconnaissable par l'effet réel qu’elle produit dans le réel. Où le réel de son effet est dans la pratique de ce qu'elle dit ou conçoit. Lorsque cette phrase est prononcée, dans ce contexte, la question est implicitement posée de savoir si la philosophie de Derrida aura toujours été plus ou moins secrètement marxiste.

Pour Rancière, les énoncés faisant effet dans le réel « reconfigurent la carte du sensible en brouillant la fonctionnalité des gestes et des rythmes adaptés au cycles naturels de la production, de la reproduction et de la soumission $\gg$. C'est ce qui les rend politiques : «l'homme est un animal politique parce qu'il est un animal littéraire, qui se laisse détourner de sa destination "naturelle" par le pouvoir des mots ». Exactement à l'endroit de son texte où il est question de définir le nœud qui tient ensemble le politique et le littéraire, la philosophie viendrait rendre le nœud qui tient l'esthétique et la politique, un nœud borroméen où la philosophie retrouverait de la place dans le domaine de l'esthétique et de la politique à la fois. À cet endroit, Rancière fait explicitement référence à la « destinerrance » et à l'écriture orpheline (voir $2000: 63$ ).

Dans un entretien de 1972 intitulé, Avoir l'oreille de la philosophie, Derrida dit quelque chose de sa pratique : « [...] consumer les signes jusqu'à la cendre, mais d'abord et plus violemment, par la verve irritée, disloquer l'unité verbale, l'intégrité de la voix, frayer ou effrayer [...] la surface calme des "mots", en soumettant leur corps à une cérémonie gymnastique [...], cérémonie à la fois joyeuse, irréligieuse et cruelle » (1972: 16). Pour Rancière, « la circulation de ces quasi-corps détermine des modifications de la perception sensible du commun, du rapport entre le commun de la langue et la distribution sensible des espaces et des occupations $\gg(2000: 63-64)$. Ces quasi-corps en circulation dessinent des « communauté aléatoires qui contribuent à la formation de collectifs d'énonciation qui remettent en question la distribution des rôles, des territoires et des langages - en bref, de ces sujets politiques qui remettent en 
Pour une théorie périphérique et/ou amoureuse : lectures d'Agamben, Derrida, Rancière

cause le partage donné du sensible » $(2000$ : 64). La description de Rancière continue de coïncider avec le projet de Glas. Comme le dit Henri Meschonnic : « Il ne s'agit pas de philologie [...]. Mais, jouant sur le "pur simulacre entre le sème et le semen", de produire un "mirage sémantique", censé dévier le vouloir-dire, établir un rapport infini des signifiants, - une forme spécifique d'associationnisme, où se dit la langue $\gg(1975: 481)$.

Si nous insistons sur Glas, c'est parce que sa parution précède d'un an le séminaire de Théorie et Pratique qui interroge la question des bords de la philosophie. Et bien que le séminaire soit la réponse au programme de l'agrégation, l'angle d'attaque choisi par Derrida est sans doute motivé par une pratique dont il vient de faire l'expérience. C'est comme si, de l'écriture de La dissémination vers celle de Glas et de La vérité en peinture, Derrida essayait une fiction de la philosophie. Cette période de fictionnalisation de la philosophie est particulièrement foisonnante dans les années 1980. Il suffit de penser à Signéponge, à ce passage parmi d'autres : «Si je préfère La Fontaine - la moindre fable - à Schopenhauer ou Hegel, je sais bien pourquoi. "Ça me paraît : $1^{\circ}$ moins fatigant, plus plaisant [...].” » (Derrida 1988 : 31).

Cela paraît à Ponge et à Derrida moins fatiguant et plus plaisant. Ponge et Derrida forment ici une communauté fictionnelle, hétérotopique jugeant le partage donné du sensible, où les critères choisis sont la fatigue et le plaisir, la propreté et les dimensions. Les petits écrits permettent de ne pas déborder, alors que la sixième séance s'ouvre avec un aveu de débordement : « Nous sommes débordés. D’où la fatigue. / Nous sommes vraiment débordés » (2017 : 121).

Seule la poésie saurait ne pas déborder. Dans l'introduction de Stanze, Agamben s'attarde sur l'héritage platonicien qui induit l'Occident à demeurer du côté de Platon dans la critique à la poésie. Celle-ci tiendrait à une scission propre au langage qui conduirait à la conclusion suivante $: \ll[. .$.$] La poésie pos-$ sède son objet sans le connaître et [...] la philosophie le connaît sans le posséder $\gg$ (Agamben $1981: 10$ ).

La poésie et la philosophie se regarderaient sur les bords d'un abîme commun, celui de la non-annexion de l'autre. La distance entre poésie et philosophie est, selon Agamben, le motif par lequel l'Occident appréhenderait l'impossibilité de saisir pleinement l'objet de la connaissance. Or la question du saisissement est la question de la possession, et la possession est intimement liée à la jouissance, qui est aussi et surtout une question de langage : c'est la raison pour laquelle les cansos des troubadours représentent le lieu à partir duquel penser le positionnement critique. 
Agamben s'inscrit ainsi dans une lignée bien connue de certains philosophes et théoriciens de la fin du XIX ${ }^{\mathrm{e}}$ et du XX $\mathrm{X}^{\mathrm{e}}$ siècles qui se sont intéressés au Moyen Âge - entre autres Friedrich Nietzsche, Denis de Rougemont, Pierre Rousselot, Anders Nygren, Jacques Lacan, Georges Bataille, Jacques Roubaud - et qui ont conçu des théories à partir d'une critique de la poésie occitane et toscane des XIII et XIV ${ }^{\mathrm{e}}$ siècles.

La théorie qui résulte de la pratique critique de Stanze pose à un certain corpus médiéval et moderne la question de savoir ce qu'est la poésie et, par effet de retour, les réponses spécifiques données par les textes autour desquels Agamben écrit, permettent à chaque fois d'établir la lisière du poétique. Autrement dit : la théorie d'Agamben est prête à être déterritorialisée.

En 1972, dans un entretien avec Michel Foucault, Gilles Deleuze affirme que les rapports entre théorie et pratique devraient être pensés de façon partielle et fragmentaire. Si d'un côté une théorie est toujours locale, relative à un domaine restreint, elle peut être appliquée dans un autre domaine et c'est justement dans cette possibilité de déterritorialisation d'une théorie que sa validité, sa pertinence et son pouvoir révolutionnaire se déploient.

Si nous appelons la théorie au sens d'Agamben, de Derrida et de Rancière une théorie périphérique, c'est que l'une des résultantes des parcours théoriques et critiques accomplis par ces intellectuels montre que la lisière $d u$ poétique tient à la faculté du poème à laisser échapper l’objet de son désir. À partir de ce constat, il serait possible d'envisager une double déterritorialisation théorique : interroger la possibilité d'employer les concepts d'amour et d'intime pour parler du rapport que le théoricien de la littérature entretient avec les textes littéraires.

Agamben fait référence à Lacan qui dans Encore dit « que l'amour vise l'être, à savoir ce qui, dans le langage, se dérobe le plus » (1975:40). En effet, dès la première séance du même séminaire, à la question de savoir « de quoi s'agit-il donc dans l'amour », ou si «l'amour, est-ce de faire un », si « l'Éros est[-il] tension vers l'Un », Lacan répond que l'expérience de la psychanalyse va à l'encontre de cela, à savoir que si le désir de faire d'l'Un est bien là, celui-ci nous conduit à nous apercevoir d'une faille. Le désir nous démontre que l'Un ne peut être atteint par le rapport sexuel parce que l'Un est dans l'essence du signifiant : «car le désir ne nous conduit qu'à la visée de la faille où se démontre que l'Un ne tient que de l'essence du signifiant ». Dans la béance de l'Un, quelque chose tient à l'être et derrière l'être à la jouissance : dans « la béance qu'il y a de cet Un à quelque chose qui tient à l'être, et, derrière l'être, à la jouissance $\gg(1975: 12)$. Plus tard, Lacan fait référence à l'amour courtois et dit : «Qu'est-ce que c'est ? C'est une façon tout à fait raffinée de suppléer à l'absence 
Pour une théorie périphérique et/ou amoureuse : lectures d'Agamben, Derrida, Rancière

de rapport sexuel en feignant que c'est nous qui y mettons obstacle. [...] Mais comment en dénoncer la feinte ? » (1975:65).

Agamben dispose les parties de Stanze comme pour répondre à cette question : celle de la dénonciation d'une feinte. Le travail critique autour des textes du Moyen Âge et du XIX ${ }^{e}$ siècle vise à formuler une définition du poétique qui en soi est une théorie de la littérature. Non seulement elle définit la fonction de la poésie, mais esquisse une définition de celle-ci comme protection de l'échappée, comme forme de l'échappement. La feinte que Lacan mentionne lorsqu'il dit « Mais comment en dénoncer la feinte ? » est ici le propre du poématique qui dispose de la place, des Stanze, pour ce qui n'est déjà plus là. Dans ce sens la poésie est dissimulation d'une absence.

Mais feinte veut aussi dire, au XIII ${ }^{\mathrm{e}}$ siècle, « fiction, invention poétique », comme dans l'usage qu'en fait Gautier de Coinci (1177-1236) lorsque pour dire le contenu du poème, il dit « sons et sonnez, fables et feintes »dans Les Miracles de Nostre Dame. Lacan bâtit toute une théorie du l'absence de rapport sexuel sur la question troubadouresque. Si le rapport sexuel n'a pas lieu en Occident, si la jouissance est dans le signifiant, c'est que la tradition poétique qui fonde l'Occident a feint cette absence, mais en retour, la poésie en langue vulgaire est née pour dire que cette absence est le tout de notre rapport au langage, le tout de notre désir comme désir de participer de la signification. Pour qu'il y ait jouissance alors, la fonction du poème est de rappeler le désir qui entraîne la tension vers la couverture de l'échancrure entre les amants et cette échancrure est aussi le bord de l'abîme sur lequel se tiennent poésie et philosophie. Ce que la poésie dans sa pratique critique vise est un supplément de jouissance. Ainsi la poésie devient-elle ce à partir de quoi définir le couple présence/absence en Occident.

Lacan vient encore en aide à Agamben au sujet de l'échancrure quand il traite de la fonction de l'écrit, encore dans le Séminaire XX: «Qu'est-ce qui se passe dans Joyce ? Le signifiant vient truffer le signifié » (1975 : 37). Que veut dire truffer? Certainement remplir, mais son sens populaire est bien tromper, comme lorsqu'on dit « c'est une trufferie », une tromperie, ou alors, « prendre quelqu'un pour une truffe $\gg$.

La pratique de la lecture montre bien qu'il existe quelque chose dans la dimension littéraire, qui intéresse les philosophes, les théoriciens et les critiques, qui n'est pas de la matérialité du texte littéraire, mais dont le texte montre la faille et qui serait dans une dimension intellectuelle, correspondant à l'immoderata cogitatio. C'est en cela que la psychanalyse définit l'Occident dans ce qu'il a à dire de l'amour, et c'est pour cela que Lacan nous invite à chérir la culture, parce que par culturel il entend ce qui du texte façonne notre cheminement vers le grand Autre. 
Une théorie périphérique et amoureuse est alors une théorie qui montre que la voie vers le grand Autre est tracée par la littérature en ceci que la fonction de l'écrit est de placer le sujet face à l'expérience d'une altérité à la fois absolument ultime, mais dont l'ultime ratage est toujours différé par le désir. C'est de l'ordre du périphérique, parce que les Stanze qui composent le poème cachent plus qu'elles n'exposent et, pour demeurer caché, il faut savoir se tenir à la marge.

\author{
Francesca Manzari \\ francesca.manzari@univ-amu.fr \\ CIELAM, Aix-Marseille Université, Aix-en-Provence \\ 29 avenue Robert Schuman \\ 13621 Aix-en-Provence \\ FRANCE
}

\title{
Références
}

Agamben, G. 1981. Stanze. Parole et fantasme dans la culture occidentale. Tr. Y. Hersant. Paris : Christian Bourgois.

Baladier, C., M. David-Menard, D. Iogna-Prat, C. Lucken. 2001. L'amour au Moyen Âge. Autour du livre de Charles Baladier. Érôs au Moyen Âge. Amour, désir et “delectatio morosa”. - Médiévales, 40, « Rome des jubilés », 133-157. https://doi. org/10.3406/medi.2001.1517

Coinci, G. de. 1966-1970. Les Miracles de Nostre Dame. Éd. F. Kœnig, 4 vol. Genève : Droz.

Derrida, J. 1972. Avoir l'oreille de la philosophie, entretien avec Lucette Finas. - La Quinzaine Littéraire, 152.

Derrida, J. 1972. Marges de la philosophie. Paris : Éditions de Minuit.

Derrida, J. 1974. Prière d'insérer. Glas. Paris : Galilée.

Derrida, J. 1988. Signéponge. Paris : Seuil.

Derrida, J. 2002. Marx \& Sons. Paris : PUF/Galilée.

Derrida, J. 2017. Théorie et pratique, Cours de l’ENS-Ulm 1975-1976. Éd. A. García Düttman. Paris : Galilée.

Foucault, M., Deleuze, G. 1972. Les intellectuels et le pouvoir. Entretien de Michel Foucault avec Gilles Deleuze. - L'Arc, 49, 3-10.

Lacan, J. 1963. Kant avec Sade. - Critique, t. XIX, 191, 291-313.

Lacan, J. 1975. Séance du 16 janvier 1973. Jacques Lacan, Le Séminaire livre XX, Encore. Paris : Seuil.

Meschonnic, H. 1975. Le signe et le poème. Paris : Gallimard.

Rancière, J. 2000. Le partage du sensible, esthétique et politique. Paris : La fabrique-Éditions. 